\title{
Quantitative analysis of 3D structures in metal-oxide composites
}

\author{
Yu Wen ${ }^{1}$, Ayako Hashimoto ${ }^{2}$, Akihiko Hirata $^{3}$ and Hideki Abe ${ }^{4}$
}

${ }^{1}$ University of Tsukuba, Tsukuba, Ibaraki, Japan, ${ }^{2}$ National Institute for Materials Science, Tsukuba, Japan, ${ }^{3}$ Waseda University, United States, ${ }^{4}$ National Institute for Materials Science, United States

Homology analysis is an efficient topological method of interpreting the shape of data [1]. Several researches have applied it to describe and classify material structures, including metallic glasses [2] and polycrystals [3]. A quantitative interpretation of structures is necessary to find correlation in the structure-property relationship, which can be accomplished using the Betti numbers, $\beta_{i}$ [4]. In the case of a two-dimensional (2D) structure image, the essential structure information, such as connectivity components and 1D holes, can be extracted and represented by $\beta_{0}$ and $\beta_{1}$, respectively. Via STEM images, we previously showed that the connectivity of the $\mathrm{CeO}_{2}$ phase, $\beta_{0}$, plays an important role in the oxygen ionic transportation of $\mathrm{Pt} \mathrm{CeO}_{2}$ composites [5]. However, this dependence does not work for the network structure, because isolated $\mathrm{CeO}_{2}$ phases might be connected three-dimensionally. Therefore, 3D structural analysis is necessary for network structures to verify the relationship between the $\mathrm{CeO}_{2}$ connectivity and ionic conductivity.

In the present study, $\mathrm{Pt} \# \mathrm{CeO}_{2}$ composites with different structures were prepared by increasing the annealing temperature from $500{ }^{\circ} \mathrm{C}$ to $800{ }^{\circ} \mathrm{C}$ under $\mathrm{CO}$ and $\mathrm{O}_{2}$ syngas. The ionic conductivities of the composites were measured using impedance spectroscopy, in the same manner as described in our previous work [5]. Focused ion beam (FIB) was used to thin the powders for observation. The STEM tomography technique was performed with a JEM-2100F (JEOL) microscope operated at $200 \mathrm{kV}$ using a high tilt specimen holder. A tilt-series was acquired over an angular range of $\pm 60^{\circ}$ with a $2^{\circ}$ tilt increment. Regarding the algorithm for $3 \mathrm{D}$ reconstruction, the weighted backprojection and simultaneous iterative reconstruction techniques were employed in DigitalMicrograph (Gatan). The generated stack slices were then binarized using the OpenCV library in Pycharm. Postprocessing including median filter, segmentation, and visualization was performed using the Avizo software (Thermo Fisher Scientific). The connectivity of the $\mathrm{CeO}_{2}$ phases, i.e., the number of $\mathrm{CeO}_{2}$ components $\left(\beta_{0}\right)$, was obtained both through the Avizo analysis and chomp calculation [6].

For temperature increments in the range of $500-700{ }^{\circ} \mathrm{C}$, the $\mathrm{Pt} \mathrm{CeO} \mathrm{O}_{2}$ composites were exhibited a lamellar structure with increasing periodicity, whereas the composites annealed at $800{ }^{\circ} \mathrm{C}$ exhibited a network-like appearance. Ionic conductivity measurements indicated that the parameters, activation energy $E$, and preexponential factor $\sigma_{0}$ increased as the annealing temperature increased. This suggests that the $\mathrm{CeO}_{2}$ connectivity was improved, and thus, $\beta_{0}$ decreased, from conclusions referred before. Figure 1 shows cross-sectional HAADF-STEM images and corresponding binary images of the $\mathrm{Pt \# CeO}_{2}$ composites annealed at $600{ }^{\circ} \mathrm{C}$ and $800{ }^{\circ} \mathrm{C}$. The black and white phases correspond to the $\mathrm{CeO}_{2}$ and $\mathrm{Pt}$ phases, respectively. Upon calculating the $\beta_{0}$ value from these $2 \mathrm{D}$ sectional binary images, the composites at $800{ }^{\circ} \mathrm{C}$ were found to exhibit the highest $\beta_{0}$ value, which is in contrast with the trends predicted via the ionic conductivity measurements. This is because, at $800{ }^{\circ} \mathrm{C}$, some separated $\mathrm{CeO}_{2}$ phases are connected at a deeper thickness, as can be confirmed by the 3D structure of the $800^{\circ} \mathrm{C}$ sample shown in Figure 2. The adjacent phases with the same color belong to the same component. From the 3D structure analysis, the $\beta_{0}$ value of the $800{ }^{\circ} \mathrm{C}$ sample was found to be lower than that of the $600{ }^{\circ} \mathrm{C}$ sample, which is inversely proportional to the ionic conductivity parameters $\left(E\right.$ and $\left.\sigma_{0}\right)$. Further $3 \mathrm{D}$ reconstructions of the $500{ }^{\circ} \mathrm{C}$ and $700{ }^{\circ} \mathrm{C}$ samples will be performed in the future. These results provide strong evidence for the relationship between the $\mathrm{CeO}_{2}$ phase connectivity and ionic conductivity of $\mathrm{Pt}^{\mathrm{C}} \mathrm{CeO}_{2}$ composites. 

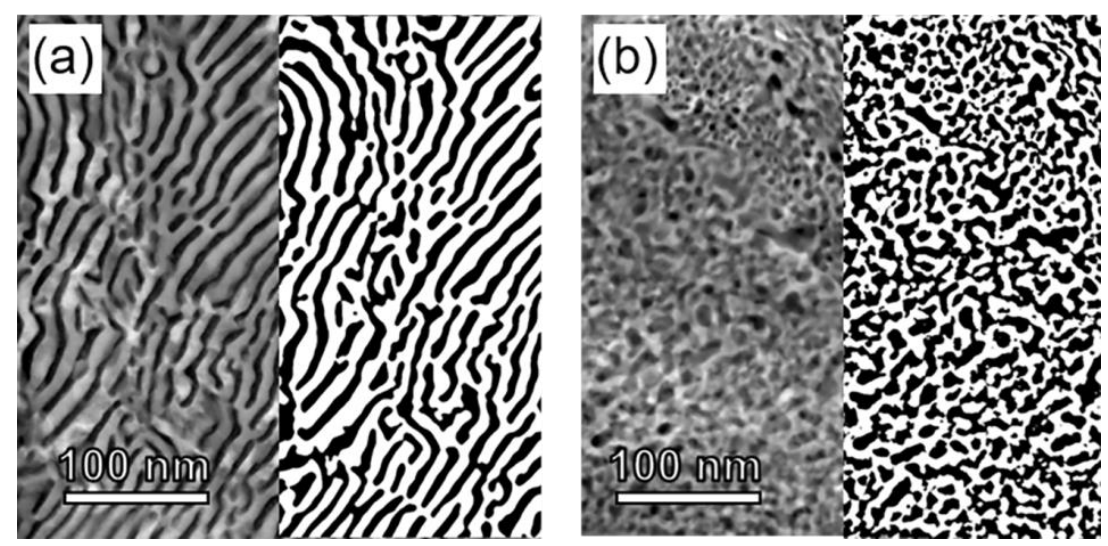

Figure 1. STEM images and corresponding binary images of $\mathrm{Pt} \# \mathrm{CeO} 2$ prepared at $600{ }^{\circ} \mathrm{C}(\mathrm{a})$ and $800{ }^{\circ} \mathrm{C}(\mathrm{b})$.

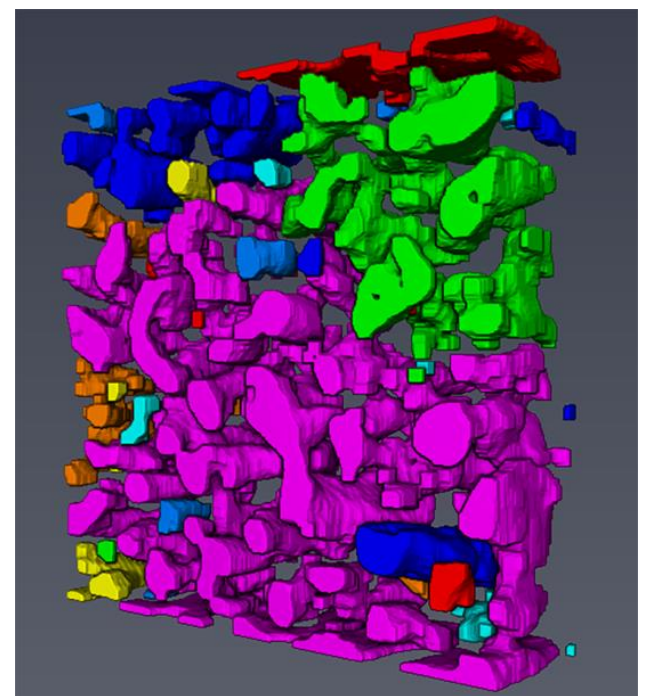

Figure 2. Reconstructed 3D image of the $\mathrm{Pt} \# \mathrm{CeO} 2$ composites annealed at $800{ }^{\circ} \mathrm{C}$.

\section{References}

[1] T. Kaczynski et al., Springer Science \& Business Media, 157 (2006).

[2] A Hirata et al., Structural analysis of metallic glasses with computational homology, Springer Japan (2016).

[3]T. Wanner et al., Acta Mater., 58102 (2010).

[4] H. Poincaré. Analysis situs, Journal de l'École Polytechnique Serie 2, 1 (1895)

[5] Y. Wen et al., Appl. Phys. Lett., 118054102 (2021).

[6] W Kalies, et al., Computational homology program, Available from: http://www.math.gatech.edu/ chom/ (2003). 